\title{
Cambios ambientales pleistoceno-holocénicos: Ocupación humana y uso de recursos en la Puna de Atacama (norte de Chile)
}

\author{
Lautaro NúÑEZ $Z^{1}$ Y Martin GrosJean ${ }^{2}$
}

\begin{abstract}
RESUMEN
Los datos paleoambientales indican que los recursos de agua, vegetación y fauna de la Puna de Atacama (>4000 m.snm), fueron abundantes durante la última edad glacial y Holoceno Temprano, mientras que las condiciones de aridez prevalecieron bajo los $3500 \mathrm{~m}$. Los cazadores del Período Arcaico Temprano (ca. 10800 a 8500 AP) estaban adaptados a la alta Puna y su piedemonte, a lo largo del desagüe de los ríos provenientes de las tierras altas, en donde los recursos de diferentes alturas se explotaban óptimamente en forma trashumante dentro de distancias cortas. Los lagos se restringieron a los niveles actuales y el proceso pedogenésico concluyó en la alta Puna. Los recursos naturales decrecieron significativamente durante el Período Arcaico Medio (ca. 8500-5000 AP), de manera que las actividades humanas se restringieron a sitios más estables en términos de recursos, entre los ríos Loa y Puripica al norte de los $23^{\circ} \mathrm{S}$. Los hábitats menos estables al sur de esta latitud (salares de Atacama y Punta Negra) fueron abandonados. Las relaciones entre puntuación de recursos, densidad ocupacional y cambios climáticos pueden ser revisados a la luz de los ajustes de los patrones de circulación subtropical, tal como se ha advertido con nuestro conjunto de datos provenientes de la Puna de Atacama.
\end{abstract}

\section{ABSTRACT}

Paleoenvironmental data from the Puna de Atacama $\left(21^{\circ}\right.$ $24^{\circ} S$ ) indicate that water, vegetation and animal resources were more abundant during late glacial and Early Holocene times than today. The rate of precipitation increased above $4000 \mathrm{~m}$ elevation to $400->500 \mathrm{~mm} / y$ r compared to the present $200 \mathrm{~mm} / \mathrm{yr}$. Dry conditions prevailed below $3500 \mathrm{~m}$. The early archaic hunters (ca. 10800-8500 BP) inhabited the High Puna and its western slope, where water was available due to higher river runoff from the altiplano, and the resources in different elevation zones were accesible. Natural resources decreased significantly during the Middle Archaic Period (ca. 8500-5000 BP). Lakes receded to today's levels, the pedogenesis in the Altiplano terminated, and human activities were restricted to the most stable sites in the rí Loa and the río Puripica catchments north of $23^{\circ} \mathrm{S}$. The less stable habitats south of $23^{\circ} \mathrm{S}$ (Salar de Atacama and Punta Negra) were abandoned. The climatic changes are best explained by shifts of the subtropical circulation.

1 Instituto de Investigaciones Arqueológicas y Museo, Universidad Católica del Norte, San Pedro de Atacama, CHILE.

2 Department of Soil Science, University of British Columbia, Vancouver, CANADA.
Las fluctuaciones climático-ambientales en los Andes Centro Sur han jugado un papel importante en las variaciones de la estabilidad ocupacional paleoindia y arcaica (Lynch 1990; Fernández et al. 1991; Núñez et al. 1993). Esto implica que los cambios en la disponibilidad de los recursos naturales impactó directamente sobre los patrones de subsistencia. Especialmente en áreas con alimentos y abastecimiento hídrico marginal, los cambios ambientales rápidos y drásticos, como los observados durante la transición Pleistoceno/Holoceno, constituyeron fuerzas conductoras primarias en el modo de subsistencia de las ocupaciones de cazadores recolectores limitadas a recursos altamente fluctuantes.

En este contexto, la Puna Salada de Atacama proporciona una excelente documentación para estudiar la interacción hombre/medioambiente desde las primeras ocupaciones arcaicas datadas hace unos 10800 años AP (Núñez 1983). En términos de clima, el área se limita hoy por un dominio extremadamente seco entre las franjas de precipitación tropical y extropical, siendo muy sensible a los cambios climáticos en donde incluso las alteraciones más sutiles vinculadas con el patrón de circulación atmosférica provocarían cambios significativos en la cantidad de agua, pedogénesis, vegetación y recursos faunísticos. La estructura vertical de los Andes y la interferencia entre el clima y la topografía produjeron fuertes gradientes con acceso a una amplia variedad de condiciones ambientales dentro de distancias verticales cortas. Esta es una clave importante para comprender la eficiencia de la trashumancia a través de circuitos estacionales entre la alta Puna y la zona de los desagües en la vertiente occidental, tanto en el pasado como hoy en día en algunas áreas relictuales de Bolivia y la región limítrofe chilena (Lauer y Erlenbach 1987).

Aunque la ocupación humana en la pendiente occidental de la Puna de Atacama es relativamente bien conocida (Lynch 1973, 1986, 1990, 1992; Núñez y Santoro 1988, 1990; Núñez 1992), las informaciones detalladas acerca de las condiciones paleoclimáticas 
vigentes en el Pleistoceno Tardío han sido más bien escasas y el contexto ambiental correlacionado con la historia se ha extrapolado de las áreas adyacentes de Bolivia, Argentina y del territorio semiárido de Chile, las que por supuesto podrían ser equívocas. Recientes estudios realizados en el área del Salar de Atacama (Figura 1) han aportado nuevos conocimientos vinculados con la dinámica de los recursos naturales en términos de variación en espacio y tiempo, con sus respectivas inferencias paleoclimáticas (Grosjean et al. 1991; Grosjean, en prensa; Messerli et al. 1993). La interrelación entre estos estudios ambientales, la secuencia ocupacional arcaica y el surgimiento de patrones de explotación de recursos son parte sustancial de un programa multidisciplinario de largo plazo actualmente en curso (Proyecto FONDECYT 1930022).

El propósito de esta primera aproximación es presentar una síntesis de las condiciones ambientales del Glacial Tardío Holoceno Temprano y discutir la relación hombre/medio ambiente en el marco del Período Arcaico Temprano (10800-8500 AP), a la luz de una estrategia multidisciplinaria geoarqueológica. El problema temporal está dado por dos preguntas claves: ¿Por qué no existen restos de ocupación paleoindia en la Puna (fase lacustre), a pesar de que existían condiciones húmedas durante el Glacial Tardío? ¿Por qué no hay continuidad de ocupación ("silencio arqueológico") en los sitios ubicados en el borde oriental del Salar de Atacama y al sur de los $23^{\circ} \mathrm{S}$ al fin del Arcaico Temprano, aunque sí hay continuación ocupacional en el río Loa, río Puripica y los oasis estables al norte de $\operatorname{los} 22^{\circ} \mathrm{S}$ ? Es necesario revisar si existieron condiciones hostiles durante el Holoceno Medio como limitantes drásticas para las ocupaciones arcaicas.

\section{Métodos}

La reconstrucción paleoambiental en este medio extremadamente árido y frío es muy difícil. Se ha puesto énfasis tanto en el control cronológico como en las correlaciones cruzadas, paralelamente a la cuantificación hidrológica, limnológica, pedológica, palinológica y glaciológica. Este mapeo de multipropósito fue realizado a diferentes alturas en la alta Puna y en el piedemonte occidental para evaluar la pendiente vertical en sus respectivos medioambientes.
La información acerca de la paleohidrografía fue obtenida de cuerpos de agua de los paleolagos. Las playas fósiles de lagos de la alta Puna fueron delineados en mapas con geodesia terrestre, correlacionados con estratos y facies de sedimentos claves debidamente fechados. Basándose en la evolución paleohidrológica lacustre, se obtuvieron escenarios paleoclimáticos y de estacionalidad, con resolución mensual (precipitación, nublados, temperatura, etc.) utilizando un modelo de balance de agua/energía (Grosjean, en prensa).

El registro de polen de ostrácodos, diatomeas en los sedimentos lacustres (Messerli et al. 1993) informó sobre la vegetación local, el transporte de polen de larga distancia y otros datos limnológicos. Los fechados radiocarbónicos en materia orgánica acuática (fracción orgánica total con técnica AMS, en carbonatos y además de fechados de termoluminiscencia en fracción polimineral 4-12 ym de capas de cenizas volcánicas y escombros de las laderas), fueron todos utilizados para el control cronológico de la evolución lacustre (Grosjean, en prensa).

La información sobre vegetación fue obtenida del registro polínico en los sedimentos lacustres y bofedales. Desafortunadamente los análisis en los depósitos de turba más antiguos no cubren el Holoceno Temprano, ni el Pleistoceno Tardío mientras que el polen en los sedimentos lacustres fue escaso. A través del análisis de paleosuelos, bofedales y turbas pertenecientes al Holoceno Temprano se observó, en términos de la descomposición física y biológica de la roca madre, la función de la situación climática y biológica. En los transectos, los factores de la pedogénesis, la roca madre, la exposición y la pendiente fueron mantenidos constantes en lo posible para que se pueda aislar el factor del clima y de la vegetación. Los paleosuelos fueron analizados en la composición total de elementos (digestión HF), Fe extraíble (CBD), CEC, C-orgánica y mineralógica de arcilla (XRD). A pesar de su bajo contenido, un horizonte Ahb subyacente o enterrado fue identificado por medio de elementos de traza que forman complejos con materia orgánica. Por otro lado, tanto la distribución espacial y el desarrollo de suelos y paleosuelos fueron mapeados en transectos vertientes localizados en la pendiente occidental de la Puna (Schreier et al. Ms).

La prospección arqueológica se estableció entre la alta Puna y el borde oriental del Salar de Atacama, 


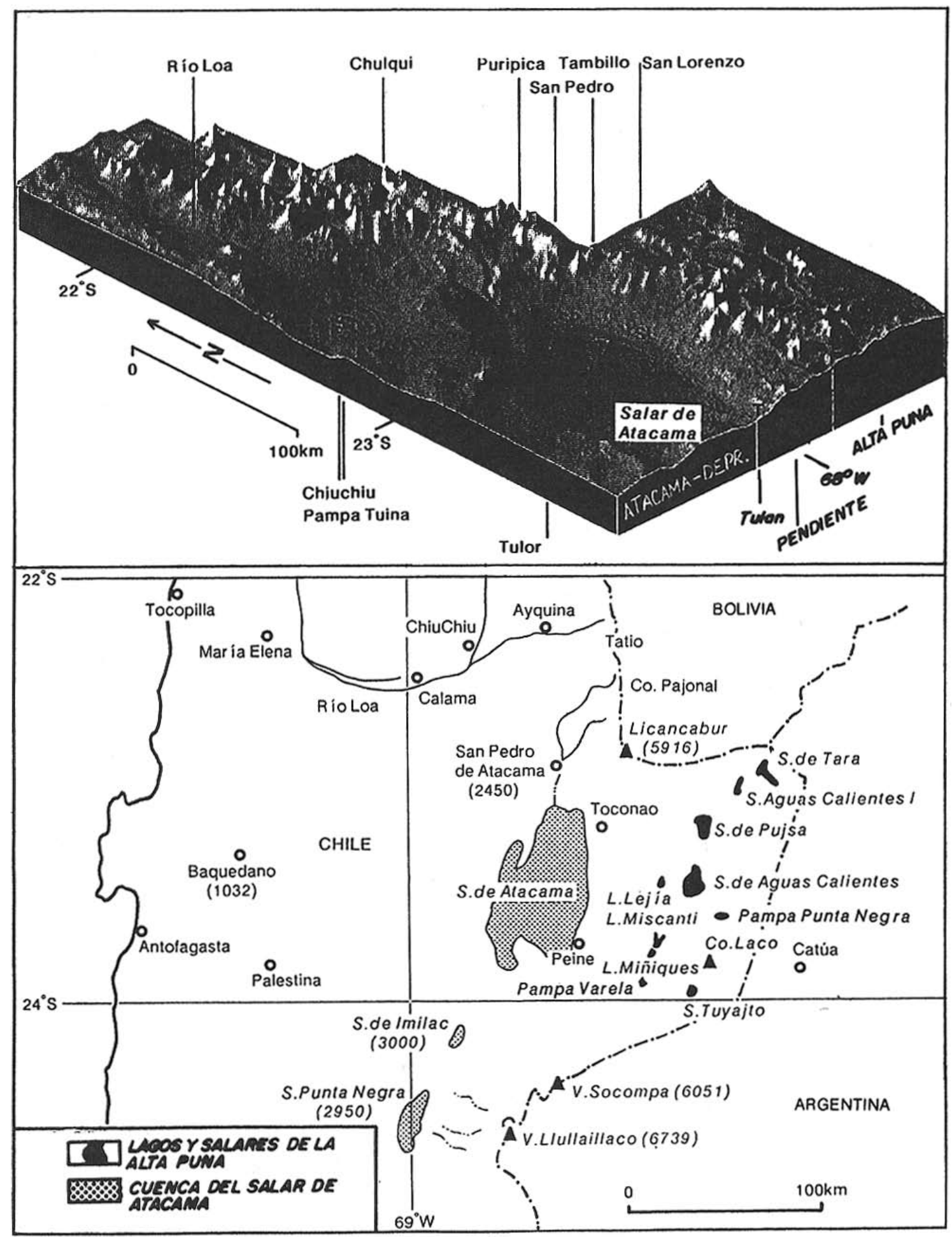

Figura 1. Area de investigación y sitios arqueológicos mencionados en el texto. 
poniendo especial énfasis en un transecto meridional a través de quebrada Tulan, un total de 11 sitios arcaicos (caza-recolección). Se fechó $\left(\mathrm{C}^{14}\right)$ cubriendo una secuencia subregional desde 10820 a 4000 años AP. Diversos análisis orgánicos, petrográficos y artefactuales dieron cuenta de las hipótesis de movilidad a nivel de interpisos de asentamientos: funcionalidad y materia de artefactos, coprolitos, depósitos de desperdicios óseos y flotación de restos orgánicos, sustentándose un conjunto de datos más confiables en términos de adaptación, movilidad y subsistencia en el flanco occidental de la Puna de Atacama.

\section{Medio ambiente del Glacial Tardío: Período Paleoindio (ca. 17000-11000 años AP)}

La edad Glacial Tardía se caracterizó por un régimen de humedad y condiciones climáticas aún frías (Figura 5). El cambio hacia el sur de la circulación tropical como lo han propuesto Kessler (1991) y Markgraf (1989) concuerda con la evidencia paleoecológica en la Puna de Atacama en términos de precipitaciones de 400-500 mm/año en las tierras altoandinas (hoy $200 \mathrm{~mm} / \mathrm{año}$ ), principalmente durante el verano (Grosjean, en prensa). Estudios recientes relacionados con la línea de nieve (Fox y Strecker 1991) y sobre paleosuelos del Glacial Tardío en Chile semiárido (Veit 1991) coinciden con las evidencias paleolimnológicas, glaciológicas y pedológicas de la Puna de Atacama a los $21^{\circ} 24^{\circ}$ (Messerli et al. 1993). Todo esto sugiere que el límite septentrional de las lluvias del invierno chileno era estable hasta $\operatorname{los} 29^{\circ} \mathrm{S}$ y no cambió hacia el norte hasta los $24^{\circ} \mathrm{S}$. Estos nuevos datos apoyan la reinterpretación de la fase lacustre detectada en el Salar de Punta Negra inmediatamente al sur del área de estudio (Lynch 1990).

Este aumento considerable de la lluvia tropical de verano y la reducida evaporación por la más alta nubosidad dio lugar a grandes lagos de aguas frescas con depósitos sedimentarios localizados sobre los $3000 \mathrm{~m}$, en un paisaje puneño actual totalmente árido, mientras que bajo los $3500 \mathrm{~m}$ no se han registrado sedimentos lacustres comparables (Salar de Atacama y de Punta Negra). Varias cuencas con altas elevaciones y patrones similar de atrapamiento como la laguna Lejía muestran similar evolución. La superficie de agua de estos lagos fue aproximadamente seis veces mayor durante el estadio máximo en relación al presente (Tabla 1). La evolución lacustre en el caso del estudio de la cuenca de la laguna Lejía se ha comparado con otras cuencas con parámetros de desagüe similares (Figura 2, Tabla 1), demostrándose efectivamente que la fase lacustre en esta laguna fue un acontecimiento regional válido para las tierras altas entre los paralelos $21-24^{\circ} \mathrm{S}$ y que estos eventos se vinculan genética y sincronizadamente con la Fase Tauca del lago Titicaca (Wirrmann y De Oliveira 1987: 315).

En efecto, en la laguna Lejía los niveles más altos (+25 m) a lo largo del último Período Glacial fueron datados después de 16715 195 años AP (HV 17806, fracción de carbonato interpretado como edad máxima) hasta después de $11700 \pm 110$ AP (ETH 6179 fracción orgánica total).

En otros lagos las playas más altas están marcadas por estramatolitos, dando edades para la fase máxima lacustre del orden de 13160 \pm 80 AP (Beta-63356/ Salar Aguas Calientes I) y $11300 \pm 70$ AP (Beta63353/Salar Ollaguie, Aguas Calientes I y laguna Lejía) durante $c a .3000$ años. La edad de la fracción orgánica total en la bentonita basal del Salar de Tuyajto (12020 \pm 95 años AP/UZ-2667/ETH-7371) confirma el significado regional de la fase lacustre de la última fase glacial.

Basados en estas evidencias advertimos que existían abundantes recursos de agua en las tierras altas de Atacama, por otro lado, extensos desagües fluviales se vertían hacia la zona basal, donde habían mesetas con aguas subterráneas y algunos cuerpos de agua abiertos en las cuencas adyacentes sobre la pendiente occidental. Esto concuerda con otros estudios anteriores vinculados con la formación de depósitos de agua subterránea durante el último glacial, tanto en la Cuenca de Atacama como en la Pampa del Tamarugal (Fritz et al. 1979). Sin embargo, no hemos detectado evidencia de este "óptimo lacustre" para aquellos grandes lagos ubicados en el piedemonte de la Puna tal como lo propone Lynch (1986).

Debido a su capacidad para almacenar agua, los glaciares han cumplido un papel importante en las fluctuaciones a corto plazo de la disponibilidad de agua. En este sentido, las dataciones directas de la expansión glacial todavía no han sido obtenidas en los Andes de Atacama. A partir de nuestras observaciones creemos que están relacionadas con condiciones húmedas bajo el influjo tropical durante tiempos tardiglaciares, de acuerdo a las siguientes consideraciones: 1) Los glaciares en ambientes permanentemente congelados como en las regiones 


\begin{tabular}{|l|c|c|c|c|c|}
\hline $\begin{array}{c}\text { Superficie del lago } \\
\left(\mathbf{k m}^{2} \mathbf{m . s n m}\right)\end{array}$ & $\begin{array}{c}\text { Lejía } \\
\mathbf{4 3 2 5}\end{array}$ & $\begin{array}{c}\text { Tuyajto } \\
\mathbf{4 0 4 0}\end{array}$ & $\begin{array}{c}\text { Miscanti/ } \\
\text { Meniques/Varela } \\
\mathbf{4 1 5 0}\end{array}$ & $\begin{array}{c}\text { Pampa Punta } \\
\text { Negra } \\
\mathbf{4 3 5 0}\end{array}$ & $\begin{array}{c}\text { Aguas Calientes I } \\
\mathbf{4 2 0 0}\end{array}$ \\
\hline Presente & 1.9 & 2.7 & 14.0 & - & $2.5-6.7$ \\
\hline Máximo Glacial Tardío & 10.8 & 14.6 & 35.6 & $<6$ & $28.8^{*}$ \\
\hline Factor & 5.7 & 5.4 & 2.3 & & $4.3-11.5$ \\
\hline
\end{tabular}

* Desagüe en el Salar Quisquiro.

Tabla 1. Superficies máximas de los lagos durante el Glacial Tardío. Datos tomados de los modelos de elevación digital (aerofotogrametría): Pampa Punta Negra (mapa topográfico 1:50000).

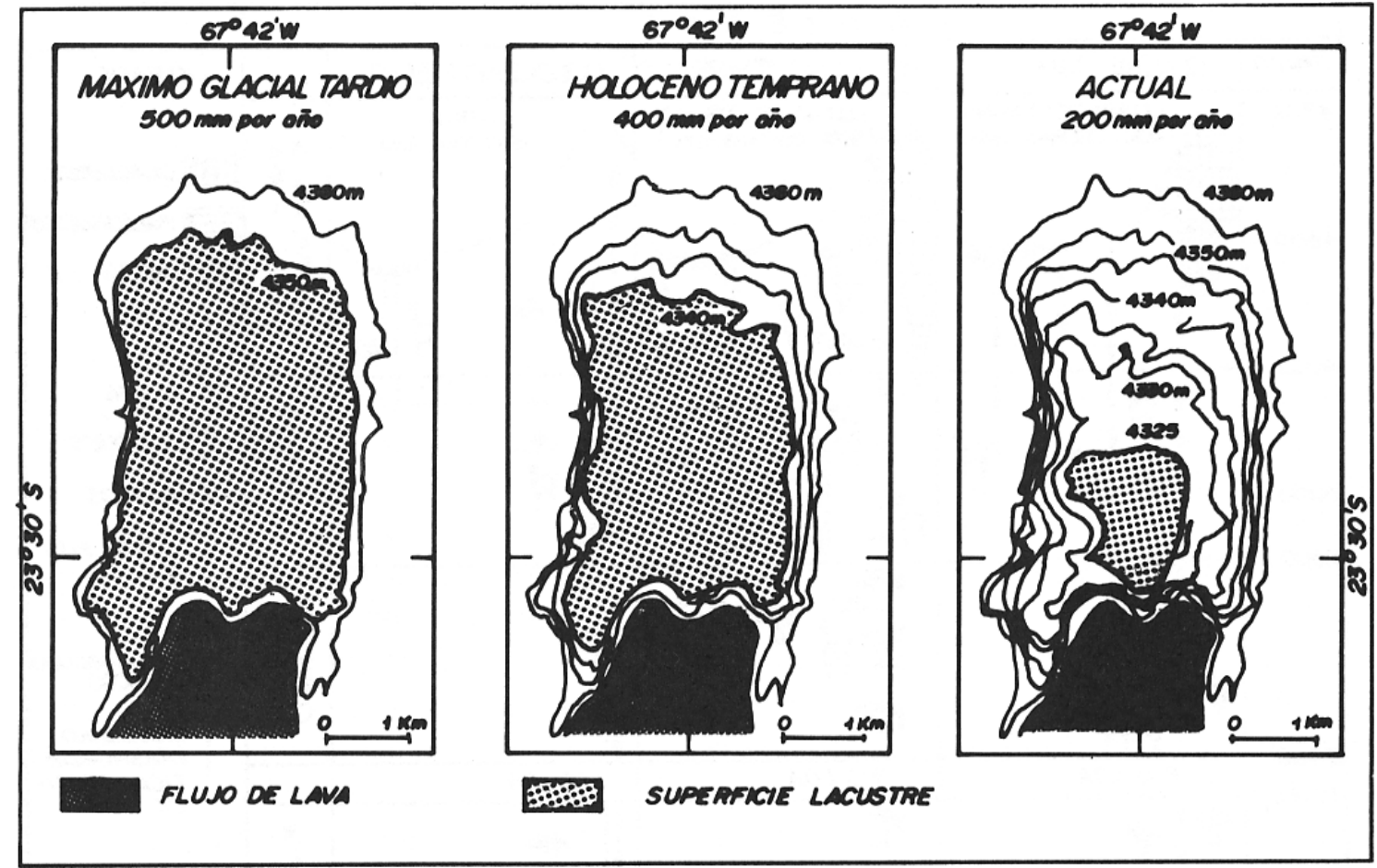

Figura 2. Evolución lacustre durante el Glacial Tardío a Holoceno en laguna Lejía (24³ 30’ S, 4350 m.snm).

altoandinas son más sensibles a cambios de precipitaciones que a cambios de temperaturas; 2) El descenso más bajo de las morrenas ecuatoriales desde $4900 \mathrm{~m}$ a los $24^{\circ} 43^{\prime} \mathrm{S}$ a $4200 \mathrm{~m}$ a los $22^{\circ} 23^{\prime} \mathrm{S}$ (Messerli et al. 1993); 3) La falta de evidencia de la expansión glacial pleistocénica en el área del Ojos del Salado a $\operatorname{los} 27^{\circ} \mathrm{S} ; 4$ ) Hay avances tardiglaciales más al norte (Clapperton 1990; Seltzer 1990).

La línea de equilibrio se localizó tan abajo como a los $4650 \mathrm{~m}$ tal como se identificó en el Cerro Pajonal a los 2230' S (Messerli et al. 1993) en el área de Atacama, sugiriendo que muchos volcanes estaban cubiertos de hielo. Este considerable volumen de agua congelado pudo proveer a las tierras bajas de agua estable adicional, siendo este un factor importante para el desarrollo de ocupaciones humanas.

La cubierta vegetacional del Glacial Tardío, como la que se indica a través del polen acumulado en los sedimentos lacustres, era escasa en las tierras altas puneñas. Se encuentra polen de Gramineae, Compositae y Chenopodiaceae en los sedimentos más tardíos (15000 AP) en el salar de Tuyajto y en la laguna Lejía (Graf 1992; Messerli et al. 1993), lo que se interpreta como una insinuación del lento aumento de las temperaturas, pero aún más que hay después del final de las condiciones globales del 
máximo frío (del registro de polen, aunque es poco, supera los registros en los sedimentos datados entre 17000 a $1500 \mathrm{AP})$.

Evidencias de condiciones frías persistentes se han localizado también en Barro Negro en la vertiente oriental de la Puna $\left(23^{\circ} 65^{\circ} 37 \mathrm{O} / 3820 \mathrm{~m}\right.$; Fernández et al. 1991). Estas temperaturas bajas fueron probablemente el constreñimiento principal para la densa vegetación del altiplano. Todavía se carece de registros vinculados con la cubierta de vegetación en la zona del piedemonte, pero respecto del ciclo regional de agua con las descargas de los ríos más altos y las mesetas con abundantes recursos de aguas subterráneas, se admite que la vegetación arbórea (Prosopis sp. y Geoffrea decorticans) en la Cuenca de Atacama fue abundante durante la última edad glacial (Ochsenius 1986). En este sentido este recurso fue un suministro sustentable para la megafauna pleistocénica aún poco conocida en el área de estudio (Tabla 2).

En relación al paleoambiente descrito, la falta de evidencia sobre ocupación humana asociada a megafauna pleistocénica en Atacama ha sido señalada (Núñez 1983; Lynch 1990; Núñez y Santoro 1990: 98); sin embargo, este sesgo puede ser errático si se tiene en mente que no existen para este período evidencias de condiciones ambientales hostiles. Así, hay concentraciones en altura de fauna pleistocénica considerable, junto a algunos artefactos líticos que podrían ser parte de la tradición paleoindia (Phillipi 1893; Casamiquela 1969-70; Núñez 1983; Ochsenius 1986; Fernández et al. 1991; Figura 4). Por otro lado, los eventos volcánicos como el descrito por Francis y colaboradores (1985), en sí mismos no parecen haber afectado a grandes áreas con posibles ocupaciones paleoindias y arcaicas, en cuanto no cubren grandes extensiones catastróficas, constatadas en el área de estudio. Al respecto, el último evento volcánico que se encuentra registrado en los sedimentos de la laguna Lejía se ha fechado por termoluminiscencia a los $16700 \pm 1200$ años AP (Bürgi 1992).

\section{Medio ambiente holocénico temprano y ocupaciones arcaicas tempranas (11000-8500 AP)}

Las condiciones de humedad prevalecieron en la alta Puna durante el Holoceno Temprano con precipitaciones de verano sobre los $400 \mathrm{~mm} / \mathrm{año}$, que duplican los $200 \mathrm{~mm} / \mathrm{año}$ actuales (Grosjean, en prensa). Se registraron niveles lacustres en el salar de Aguas Calientes I, donde se ubicaron carbonatos laminados que estaban siendo precipitados después de los 10400 \pm 75 años AP (fracción carbonato interpretada como edad máxima/efecto reservorio. Además, el conjunto de diatomeas en los sedimentos de la laguna Lejía sugiere un incremento de las aguas

\begin{tabular}{|c|c|c|c|}
\hline Sitios & Tiempo (AP) & Especies & Referencias \\
\hline Pampa de Tamarugal & $\begin{array}{l}\text { Pleistoceno } \\
\text { Tardío }\end{array}$ & $\begin{array}{l}\text { Megatherium } \\
\text { Scelidon } \\
\text { Macroauquenia } \\
\text { Equus }\end{array}$ & $\begin{array}{l}\text { Casamiquela 1969-70 } \\
\text { Casamiquela 1969-70 } \\
\text { Casamiquela 1969-70 } \\
\text { Casamiquela 1969-70 }\end{array}$ \\
\hline Barro Negro (3820 m) & $\begin{array}{l}12530 \pm 160 \\
10200 \pm 140\end{array}$ & $\begin{array}{l}\text { Hippidion sp. } \\
\text { Hippidion sp. }\end{array}$ & $\begin{array}{l}\text { Fernández et al. } 1991 \\
\text { Fernández et al. } 1991\end{array}$ \\
\hline Quereo & $11400 \pm 155$ & $\begin{array}{l}\text { Mastodonte Cuvieronius sp. } \\
\text { Equus sp. } \\
\text { Ciervo (Antifer sp.) } \\
\text { Camelidae } \\
\text { Mylodon sp. }\end{array}$ & $\begin{array}{l}\text { Núñez et al. en prensa } \\
\text { Núñez et al. en prensa } \\
\text { Núñez } \text { et al. en prensa } \\
\text { Núñez et al. en prensa }\end{array}$ \\
\hline Tagua Tagua & $\begin{array}{l}11000+170 \\
10120 \pm 130\end{array}$ & $\begin{array}{l}\text { Mastodonte, Equus } \\
\text { Ciervo (Antifer sp.) } \\
\text { Mastodonte (C. humboldti) }\end{array}$ & $\begin{array}{l}\text { Montané } 1972 \\
\text { Montané } 1972 \\
\text { Núñez et al. } 1992\end{array}$ \\
\hline
\end{tabular}

Tabla 2. Fauna pleistocénica del centro-norte de Chile y áreas aledañas. 
salinas asociadas a alta evaporación y largo tiempo de procesos de enriquecimiento en las aún grandes extensiones de cuerpos de agua.

Un paleosuelo del Holoceno Temprano ubicado entre los 4000 y $4800 \mathrm{~m}$ se ha interpretado como una evidencia adicional para el desarrollo de condiciones húmedas y estables en la alta Puna. El paleosuelo sobre los 4000 m muestra una concentración más alta de hierro activo o poco cristalino $\mathrm{Fe}_{\text {C-D }}$ una arcilla mineralógicamente más diferenciados además de un desarrollo más avanzado de horizontes de suelos IIA $_{\mathrm{HO}}$ y IIB $_{\mathrm{i}}$ y un muy débil horizonte $\mathrm{A}_{\mathrm{h} . \mathrm{b}}$. Esto indica una antigua presencia de vegetación densa y considerable actividad biológica y física (Messerli et al. 1993; Schreier et al. Ms).

Los mejores paleosuelos se constituyeron por los $4500 \mathrm{~m}$, unos $500 \mathrm{~m}$ más arriba del curso de la línea de máxima vegetación actual. Dado el control de humedad del límite inferior del cinturón vegetacional, el control de temperatura del límite superior del cinturón referido, y una gradiente de temperatura de $0.7^{\circ} \mathrm{C} / 100 \mathrm{~m}$, sugerimos en consecuencia el desarrollo de veranos de temperatura incrementada alrededor de $3.5^{\circ} \mathrm{C}$. En relación a la edad del paleosuelo sólo una datación indirecta es disponible y suficientemente auspiciosa: su pedogénesis es postglacial, terminada antes de los 7400 años AP (Schreier et al. Ms). Además, el Horizonte II Bm está relacionado al más alto nivel del aterrazamiento lacustre del Holoceno Temprano, asociado con artefactos líticos triangulares del Período Arcaico Temprano (Figuras 3 y 6). Así, concluimos que su edad pedogenética corresponde al Holoceno Temprano. También la distribución vertical de estos suelos indica que en las regiones altoandinas como éstas existían condiciones ambientales óptimas. Esto coincide con la formación de aguas subterráneas durante el Holoceno Temprano, tal como se ha reportado para el área de San Pedro (Fritz et al. 1979: 540).

A pesar de las condiciones cálidas y húmedas, las concentraciones boscosas se localizaron en el borde oriental de la cuenca de Atacama, generándose hábitats alternativos en los desagües de los arroyos y planicies de bosques de algarrobos y chañares.

Hasta ahora las primeras ocupaciones humanas detectadas en la Subárea Circumpuneña son de naturaleza arcaica temprana correlacionadas con eventos holocénicos tempranos (ver Figura 5), en estricta asociación a un escenario paleogeográfico dominado por los recursos de lagos altos, actualmente secos o con espejos sensiblemente restringidos.

En efecto, las evidencias en la cuenca de la laguna Lejía señalan que las condiciones húmedas prevalecieron en las tierras altas durante el Holoceno Temprano con precipitaciones de verano de hasta $400 \mathrm{~mm} / \mathrm{año}$ (Grosjean, en prensa). Los restos de estromalitos marcan los niveles del lago $(c a+10 \mathrm{~m})$, correspondiente: tres veces la superficie actual, a una superficie lacustre total del orden de $6600 \mathrm{~km}^{2}$. Entre los 11000 y 8500 años AP los lagos presentaron más y más salinidad tal como lo indican los cambios de los conjuntos de diatomeas. La edad de una capa de arena orgánica en el salar de Aguas Calientes I (8430 75 años AP, fracción orgánica total, ETH-5846), indica la edad mínima para el final de la fase lacustre en esta cuenca. Aunque no existe una información precisa sobre la desaparición de los lagos puneños, el lapso de 8000-7500 años AP es coherente y se apoya en la terminación de la formación del suelo durante el Holoceno Temprano (Schreier y Grosjean Ms) y en los bajos niveles del lago en la cuenca del Titicaca, después de los 7700 años AP (Wirrmann y De Oliveira 1987). La condición cálida/húmeda del Holoceno Temprano parece estar ligada otra vez al cambio hacia el sur de la circulación tropical, la que probablemente interactuaba con el cambio hacia el sur de la provincia Panamic en el Pacífico del este (Rollins et al. 1986), lo que trajo consigo aguas tropicales cálidas al área de Antofagasta $\left(22^{\circ} \mathrm{S}\right)$, según las evidencias constatadas a través de la identificación de otolitos de peces tropicales intrusivos y no localmente extintos, registrados en la cercanía de Antofagasta (Llagostera 1979).

Como se ha señalado, en términos de uso de recursos las condiciones ambientales estables y favorables en las tierras altas puneñas se demuestran mejor con la formación de un paleosuelo durante el Holoceno Temprano entre los 3500 y $4800 \mathrm{~m}$.snm. Aunque la formación del suelo es generalmente débil (condiciones cálidas y húmedas duraron sólo 2000 a 3000 años) y la subsecuente alteración periglacial y solifluidal del paleosuelo, se ha registrado un horizonte orgánico $\left(A_{h b}\right)$, cubierto en tiempos anteriores en los sectores bien protegidos de la cima de las morrenas, indicando densa vegetación y considerable actividad biológica (Schreier y Grosjean Ms). El límite inferior de humedad controlada a $4700 \mathrm{~m}$ y el desarrollo máximo del suelo $c a$. $500 \mathrm{~m}$ sobre el 


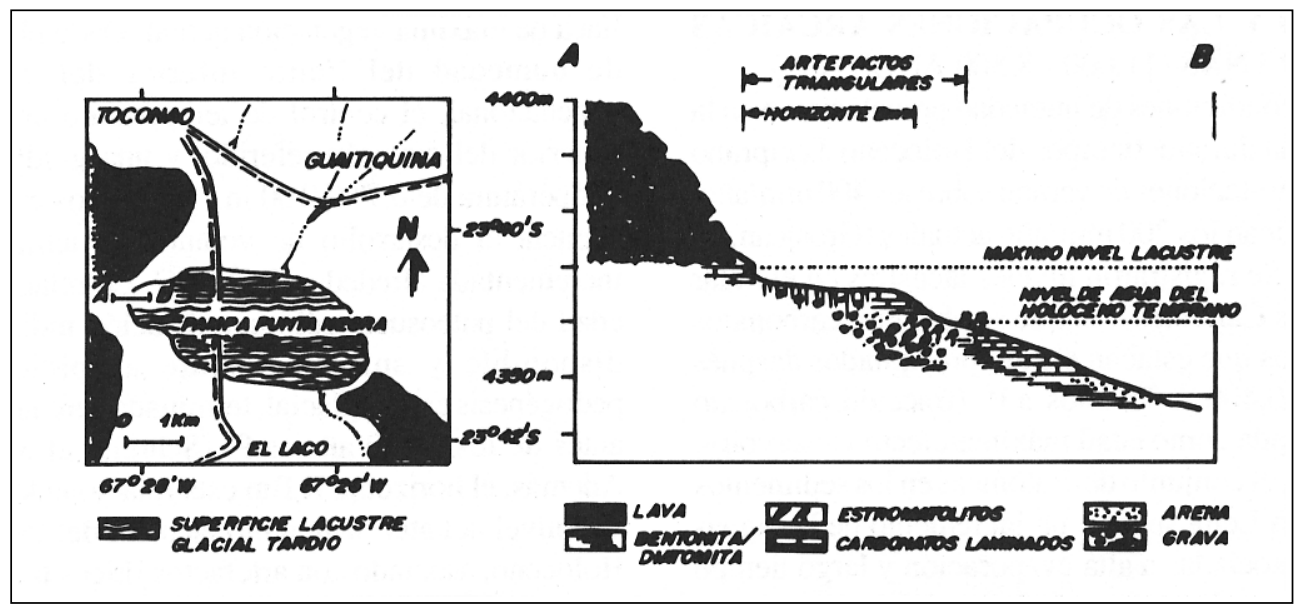

Figura 3. El lago Pampa Punta Negra durante el Glacial Tardío-Holoceno Temprano y su perfil seccional con paleosuelo Bm y artefactos líticos triangulares del Período Arcaico Temprano depositados en la terraza del Glacial Tardío.

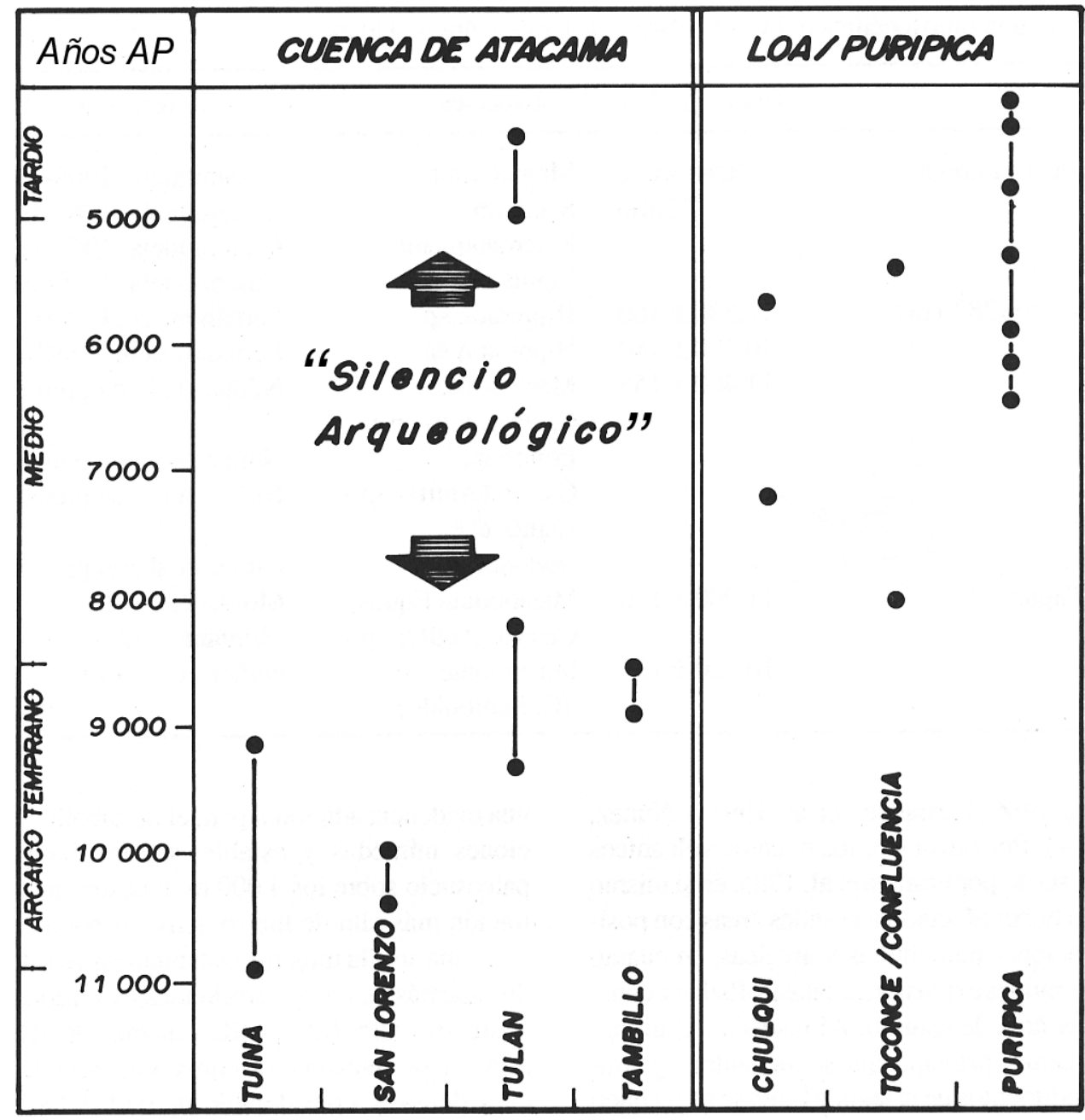

Figura 4. Secuencia cronoestratigráfica de los sitios arqueológicos arcaicos en la Cuenca de Atacama y río Loa, datados entre 11000 y 4000 años AP. 


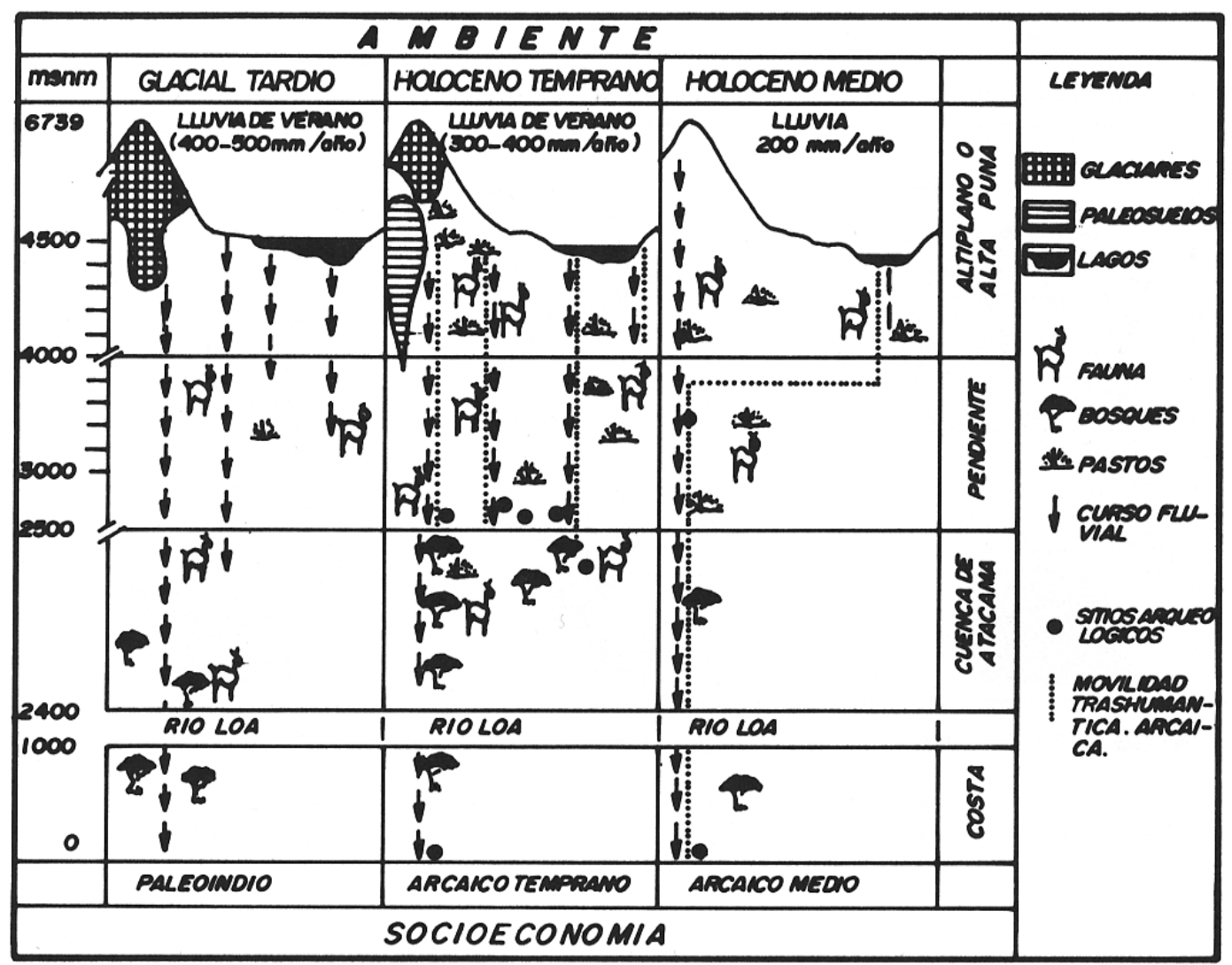

Figura 5. Hipótesis de reconstrucción ambiental y ocupación humana en la vertiente occidental de la Puna de Atacama durante el Glacial Tardío y el Holoceno Medio $\left(22-23^{\circ} \mathrm{S}\right)$.

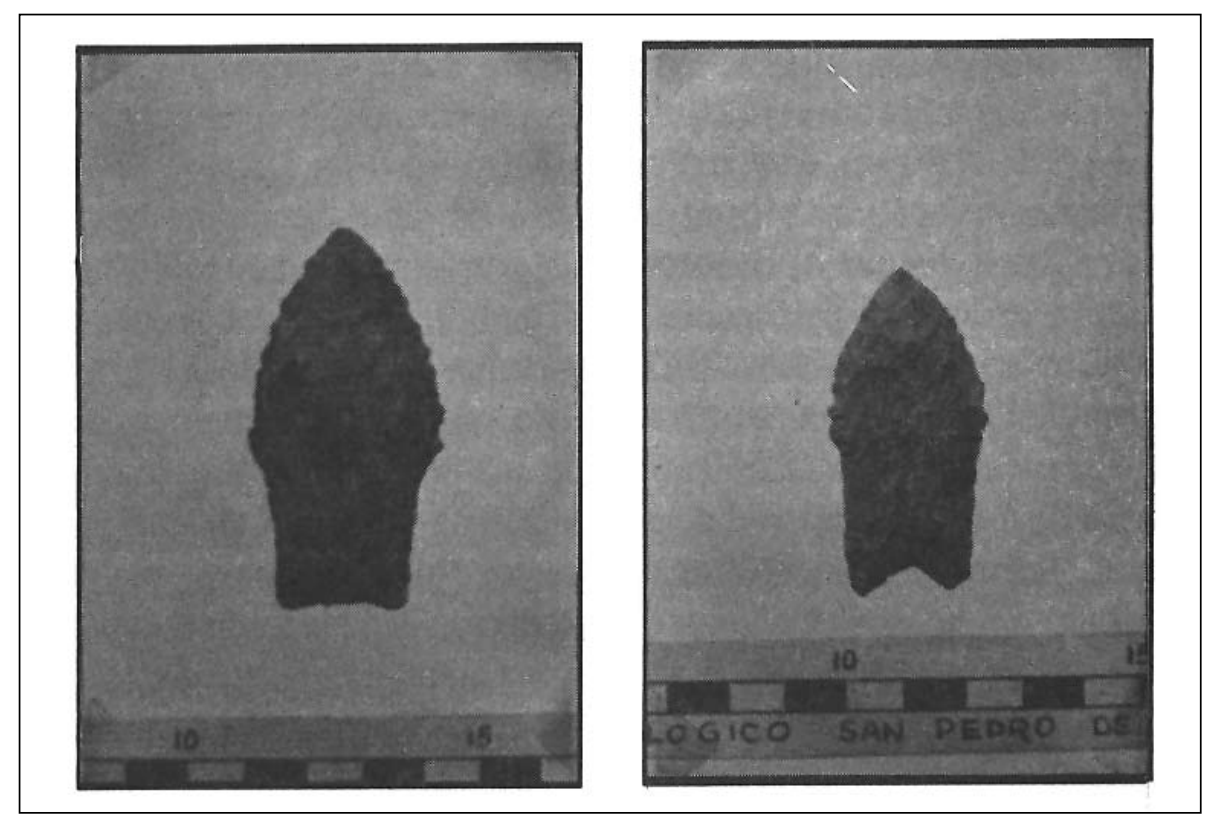

Figura 6. Puntas basálticas y bifaciales de forma eventualmente paleoindio registradas en la superficie del piso altiplánico en el sitio Potrero (frontera chileno-boliviana). 
máximo común de vegetación a 4500-4600 m dan cuenta de los $3.5^{\circ} \mathrm{C}$ de temperaturas más cálidas (verano) y las precipitaciones de verano durante el Holoceno Temprano. La formación de este paleosuelo postglacial ocurrió al final, anterior a $c a .7400$ años AP (Messerli et al. Ms), probablemente contemporáneo al descenso de los niveles lacustres $(8500$ años AP). En este sentido, la distribución vertical de estos suelos enfatiza que las tierras altas puneñas eran fuentes de ríos con desagües que sustentaban el suministro de agua para el nivel de base regional o piemontana, situada en la pendiente occidental, donde la formación de aguas subterráneas se ha reportado en los oasis y planicies aledañas localizadas bajo los $2300 \mathrm{~m} . \mathrm{snm}$.

En este paisaje la evidencia cronoestratigráfica más temprana de ocupación humana arcaica se ha registrado consistentemente en Tuina 1 (10800 AP), San Lorenzo 1 (10400 AP), Chulqui 1 (9600 AP), Tulan 68 (9300 AP) y Tambillo 1 (8600 AP y otra datación reciente de $8870 \pm 70$; Beta-63365) y otras del salar de Punta Negra (Sinclaire 1985; Núñez y Santoro 1988; Lynch 1990; Lynch y Stevenson 1992; Núñez 1992).

La distribución espacial de estos sitios (ver Figura 1) muestra vínculos con ricos paleorrecursos en la alta Puna, en cuyas cuencas lacustres se han ubicado similares artefactos líticos debido a la interacción trashumántica que existía a lo largo de los desagües entre las tierras altas, el piedemonte y quebradas, con recursos más estables de agua, vegetación y concentraciones de avifauna. Las lluvias de verano y las temperaturas más cálidas fueron favorables para la concentración de aves y herbívoros y, consecuentemente, las tierras altas puneñas entregaron las mejores condiciones para la cacería estacional postglacial de camélidos, aves y roedores, mientras que los desagües fluviales y los suministros de aguas subterráneas entregaron espacios vitales en términos de recolección vegetal y cacería a lo largo del piedemonte durante el invierno (quebradas y oasis bajos). Todavía no existen fechados radiocarbónicos disponibles para la alta Puna, pero la asociación de los niveles lacustres del Glacial Tardío/Holoceno Temprano y la formación de suelos (II Bm), con artefactos líticos triangulares del patrón Tambillo, bajo el espejo de agua holocénico temprano en las playas altas expuestas durante este Período Arcaico Temprano. El mismo patrón se advierte en los lagos de Aguas Calientes I, Tara, Lejía y Pampa Punta Negra (cuenca seca actual), modificado en las cuencas de Tuyajto, Meniques y Miscanti (ver Figura 3).

El registro de similares hojas basálticas y de obsidiana del patrón triangular holocénico temprano (Tuina/ Tambillo), tanto en Aguas Calientes I (4100 m) como en Tambillo 1 ( $2400 \mathrm{~m}$ ), son evidencias de movilidad vertical estacional, con traslados de materia prima lítica a través de los transectos alta Puna-Salar de Atacama, articulándose áreas de recursos dinámicos (caza y recolección) con concentraciones de dependencia estacional.

Estas ocupaciones son sincrónicas y coparticipantes de similares circuitos trashumánticos circumpuneños de acuerdo a las evidencias trasandinas de Inca Cueva y Huachichocana (Fernández Distel 1974; Aschero 1983), incluyendo similares patrones residenciales (abrigos rocosos) y alimenticios (Yacobaccio 1983). En estos sitios sólo hay evidencias de fauna moderna (dominio de camélidos), habiéndose extinguido la propiamente pleistocénica a raíz de los drásticos cambios de vegetación al inicio del postglacial, tal como ocurrió en Barro Negro cerca de Jujuy (Fernández et al. 1991).

\section{Medio ambiente holocénico y ocupaciones humanas (silencio arqueológico): 8500-5000 años AP}

Los grandes lagos como Aguas Calientes I se secaron más o menos por los 8400 años AP (Grosjean Ms) y la formación de los suelos terminó antes de los 7400 años AP (Schreier et al. Ms). Estos eventos indican un cambio significativo hacia condiciones más secas, con disminución de agua, vegetación y recursos faunísticos hacia el optimum thermal, más o menos por los 6000 años AP.

Indicadores para el establecimiento de climas áridos a gran escala conectados al Pacífico del este han sido mencionados por Enfield (1989) para el Período Arcaico Medio y por Rollins y colaboradores (1986) para ocupaciones registradas aproximadamente por los 5000 años AP. Evidencias sobre recursos inestables y altamente variables hemos localizado en los sedimentos holocénicos de la quebrada Puripica, en donde una secuencia de ocupaciones humanas (fogones y artefactos) pertenecientes al Arcaico Medio se han datado entre $6420 \pm 230$ AP (Beta-63366) y $5300 \pm 100$ AP (Beta-63360). Estos restos de pisos de campamentos abiertos se encuentran encajados en sedimentos clásticos y (fluviales/torrenciales) que 
representan crecidas extraordinarias o eventos de tormentas aisladas. Tales sucesos se correlacionan con lluvias torrenciales que pueden haber causado inundaciones temporales incluso en el más árido Salar de Atacama como lo demuestran los depósitos de arcillas/aluvio en las capas de arenas datadas a los $5605 \pm 65$ años AP $\left(\mathrm{C}^{14}\right.$ en el aluvión a $150 \mathrm{~cm}$ ETH-5848) localizado en un perfil de sedimentos expuestos entre San Pedro y Tulor.

La tendencia a la aridez creciente hacia el optimum thermal se reitera en sitios como Quereo (Núñez et al. 1993) y Barro Negro en el Noroeste Argentino (Fernández et al. 1991), incluido el lago Titicaca (Wirrmann y De Oliveira 1987). Por lo tanto, parece ser un fenómeno regional probablemente ligado a una desviación hacia el norte de la circulación del verano (sub)tropical y una creciente influencia del anticiclón del sureste del Pacífico (SPH), sumado a la sobreimposición de la corriente de Humboldt (Rollins et al. 1986; Enfield 1989).

Resulta de interés conocer con más detalles cuál fue el impacto del cambio de las condiciones medioambientales en las estrategias de subsistencia y equipamiento tecnológico de las ocupaciones arcaicas tempranas del Holoceno Temprano, en términos de ajustes readaptativos frente a un proceso de crisis de recursos delimitado entre 8500-4800 AP. En este tiempo precisamente los inicios de las prácticas agropastoralistas circumpuneñas se correlacionan con innovadores regímenes de producción de alimentos. Se sabe que el proceso creciente de aridez afectó tanto a la vertiente oriental de la Puna como a la occidental, constituyéndose aquí un "silencio arqueológico" al sur de los $23^{\circ} \mathrm{S}$ por los $8000-5500$ años AP, precisamente cuando debieron lograrse los primeros inicios readaptativos pecuarios y agrícolas (Núñez 1983; Yacobaccio 1983).

Durante la aparición de condiciones áridas algunos asentamientos arcaicos tempranos asociados a recursos más potenciales (p.e., ríos Loa, Puripica, etc.) pudieron persistir, mientras que otros localizados en ambientes más frágiles se movilizaron dando lugar a ciertos "silencios arqueológicos" (cuencas de Atacama y Punta Negra) detectados en las secuencias regionales.

En este contexto ecológico los hábitats del Período Arcaico Medio se localizan en enclaves con recursos más confiables asociados a arroyos y vertientes bajo los $4000 \mathrm{~m} . \mathrm{snm}$, acentuándose el régimen trashumántico de caza-recolección especializado en el aprovechamiento de la diversidad estacional y altimétrica (estadio Puripica).

Este régimen de movilidad hacia enclaves con menos stress ambiental pudo incluir el mayor poblamiento del litoral desértico por un lado (Núñez 1992), paralelo a la búsqueda de nuevas opciones productivas. En este sentido, hay evidencias de que en esta época comenzaron las prácticas regionales de domesticación de camélidos (Hesse 1982a y b; Núñez 1983, 1989, 1992; Yacobaccio 1983) y la primera experimentación hortícola y agrícola en las postrimerías del Arcaico. Estas nuevas orientaciones económicas tenderían a lograr más autonomía en relación a la disminución de recursos apropiatorios de caza y recolección a raíz del aumento de las fluctuaciones de corto y mediano plazo en términos de agua, vegetación y suministro de recursos cárneos.

Las primeras fuerzas de tareas dedicadas al pastoralismo y cultivación de la tierra en un contexto de tradiciones de caza y recolección arcaica alcanzaron el desarrollo de economías de reserva, con bodegaje y mantención de alimentos para las etapas de escasez. Esta estrategia adaptativa dio lugar al surgimiento $a$ posteriori de sociedades complejas agrocerámicas adecuadas a las condiciones áridas de la Puna de Atacama (tradición atacameña).

El reajuste adaptativo más exitoso se basó en la crianza de camélidos cuando los regímenes de precipitaciones disminuyeron, junto a la emergencia de tecnologías orientadas a la reducción de riesgos de subsistencia, paralelo a adaptaciones sociales que combinan las prácticas de caza con pastoralismo.

La historia climática regional en este período, aunque muy parcialmente conocida, presenta indicios de oscilaciones imprevisibles que provocan desajustes en la reproducción de las manadas salvajes: sequías alternadas de eventos con depósitos fluviales torrenciales detectados en la quebrada de Puripica. Precisamente, los estudios de Druss (1977); Hesse (1982b) y Núñez (1983) y han explicado que en ambientes áridos los cambios del régimen de humedad producen efectos catastróficos en los recursos de caza, estimulando la emergencia de pastoralismo circumpuneño entre 4800 a 4000 AP. 


\section{Conclusiones y perspectivas}

Nuevas evidencias en torno a las condiciones paleoambientales de la Puna de Atacama ponen énfasis en la explotación vertical y estacional de los recursos hídricos, vegetacionales y avifaunísticos a través de circuitos trashumánticos entre las tierras altas y medias durante el Glacial Tardío y el Holoceno Temprano. Mientras el aumento de las precipitaciones de verano estaban espacialmente limitadas a la alta Puna, en el piedemonte adyacente se consolidaron espacios vitales en los desagües de los ríos, vertientes y sectores con acumulación de aguas subterráneas derivadas de las tierras altas. Esta interacción entre tierras altas y bajas, incluyendo varias otras franjas altitudinales intermedias, fue fundamental para consolidar el régimen trashumántico de los grupos cazadores recolectores durante el Arcaico Temprano. Estos se establecieron en el transecto a través de una estrategia adaptativa con acceso a diferentes zonas-pisos, dentro de distancias de traslado vertical relativamente cortas, utilizando eficientemente la diversidad estacional del ciclo anual.

Se acepta que los recursos de agua y de animales eran abundantes y estaban disponibles al final del Pleistoceno (Período Paleoindio), pero no hemos detectado ocupaciones específicas a pesar de que los paleoambientes no eran hostiles inmediatamente antes del Holoceno. Al respecto, se han detectado sólo dos artefactos líticos en superficie por Le Paige (com. pers.), en las playas de una depresión llamada Potrero en la frontera chileno-boliviana. Estos resultan ser distintos al total de las colecciones líticas del área. Ambos fueron observados por J. Bird (com. pers.) y corresponderían tipológicamente a las formas finipleistocénicas que se podrían vincular con las tierras altas del área (Figura 6).

Las ocupaciones arcaicas medias se vincularon con etapas de mayor stress ambiental reflejado en un "silencio arqueológico" localizado, tanto en el Salar de Atacama como en el más meridional de Punta Negra. Sin embargo, en la sección septentrional, entre el río Puripica y el Loa, incluyendo Toconce y buena parte del sur peruano (Aldenderfer 1988) las ocupaciones arcaicas no presentan discontinuidad. En estos casos se detectan circuitos trashumánticos entre ambientes tan contrastados como aquellos sitios correlacionados de Chiu Chiu (2400 m), Puripica (3400 m) y la alta Puna de Puripica (4000 m), en los que ocurrió la domesticación de camélidos por los 4600-4800 años AP, paralela al manejo inicial de horticultura en la vertiente trasandina.

Estas innovaciones socioeconómicas habrían gatillado un proceso de experimentación de nuevos patrones de explotación de recursos, más independiente de las fluctuaciones ambientales de corto tiempo. La disminución de los recursos naturales pudo conducir a nuevas transformaciones al interior de la sociedad cazadora recolectora del Arcaico Temprano. Las nuevas labores pastoriles, horticultoras y artesanales complejas (p.e., metalurgia, cerámica y textiles) datadas por los 3000-3500 años AP sugieren que los cambios en este período se comprometieron con la emergencia de sociedades más complejas (Fase Tilocalar).

La naturaleza de los cambios climáticos locales guardaría relación con la circulación monzónica en áreas subtropicales. En efecto, las precipitaciones del cinturón tropical se trasladaron al sur cerca de $8^{\circ}$ a $10^{\circ}$ de latitud. Por ejemplo, la isoyeta de $400 \mathrm{~mm} /$ año, la cual está presente ahora en el lago Titicaca $\left(c a .16^{\circ} \mathrm{S}\right)$, se movió tan lejos como los $24^{\circ} \mathrm{S}$ durante el Glacial Tardío y el Holoceno Temprano. Este evento creó las condiciones de humedad en la alta Puna, fijándose los espacios de recursos vitales para el acceso de cazadores recolectores arcaicos tempranos. De ser así, la restricción de la circulación monzónica pudo generar condiciones de aridez en la porción más frágil de la Puna Salada, dando lugar a cuadros de inestabilidad o "silencios arqueológicos" que merecen mayor investigación, toda vez que los recursos de subsistencia humana y animal son muy sensibles a los cambios climáticos en regiones con ecosistemas áridos. A diferencia de las latitudes más altas, aquí los cambios de temperaturas y lluvias fueron más dramáticos. Por ahora, los datos disponibles provienen de distintas disciplinas y sostienen que los cambios ambientales ocurrieron y aquí vivían comunidades humanas que debieron responder con estrategias creativas para mantener sus asentamientos en un contexto de críticas transformaciones ambientales. Estas investigaciones geoarqueológicas continúan con estas orientaciones a través de tres transectos en las tierras altas $\left(18^{\circ} \mathrm{S}, 24^{\circ} \mathrm{S}\right.$ y $\left.27^{\circ} \mathrm{S}\right)$, para la mejor comprensión de los procesos de interacción entre respuestas humanas y mecanismos de cambios climáticos y ambientales en la más inhóspita Puna Salada de los Andes Centro Sur. 
Agradecimientos Este artículo es una primera síntesis del Proyecto (NF 21-27/824.89) de la Fundación Nacional de Ciencias de Suiza donde participa el segundo autor. Integra a su vez la problemática general y primeros resultados del Proyecto FONDECYT 1930022 "Reconstitución multidisciplinaria de eventos culturales y ambientales pleistocénicos-holocénicos de la quebrada de Puripica (Puna de Atacama)", conducido por el primer autor en asociación a Martín Grosjean y a Juan Varela del Depto. de Geología de la Universidad de Chile. Este trabajo ha integrado los principales datos del artículo "Late glacial Early and Middle Holocene environments human occupation and resource use in Atacama (Northern, Chile)" de Grosjean y Núñez a publicarse en Geoarchaeology. Estamos muy agradecidos de los miembros del equipo multidisciplinario de la Misión Suiza, quienes con sus resultados han contribuido sustancialmente a hacer posible esta propuesta de síntesis y nuevas expectativas de investigación: B. Messerli, M. A. Geyh, K. Graf, K. Ramseyer, V. Schotteter, H. Schreier y M. Vuille. Un agradecimiento especial a Sylvia Welke por su asistencia en terreno, incluyendo a Willi Egli, Hugo Romero y Marcela Espinoza (DIFROL) por la ayuda logística, permisos de investigación y toda clase de provisiones.

\section{REFERENCIAS CITADAS}

ALDENDERFER, M., 1988. Middle Archaic Period domestic architecture from Southern Peru. Science 241:1828.

ASCHERO, C., 1983. El Sitio ICc-4: Un asentamiento precerámico en la quebrada de Inca Cueva (Jujuy, Argentina). Estudios Atacameños 7: 62-72.

BÜRGI, A., 1992. Aufbau und Betrieb eines ThermolumineszenzLabors zur. Datierung quartärgeschichtlicher Proben. Ph. D. Dissertation, University of Bern, Berna.

CASAMIQUELA, R., 1969-70. Primeros documentos de la paleontología de vertebrados para un esquema estratigráfico y zoogeográfico del Pleistoceno de Chile. Boletín Prehistoria de Chile 2-3 (2): 65-73.

CLAPPERTON, C., 1990. Quaternary glaciations in the Southern Hemisphere. Quaternary Science Review 9: 121-304.

DRUSS, M., 1977. Computer analysis of Chiu Chiu Complex settlement pattern. El Dorado 1 (3): 51-73.

ENFIELD, D. B., 1989. El Niño, past and present. Review of Geophysics 27 (1): 159-187.

FERNANDEZ, J., V. MARKGRAF, M. PANARELLO, F. ANGOLINI, S. VALENCIO, y M. ARRIAGADA, 1991. Late Pleistocene/Early Holocene environments and climates, fauna and human occupation in the argentine altiplano. Geoarchaeology.

FERNANDEZ DISTEL, A., 1974. Excavaciones en las cuevas de Huachichocana, Depto. de Tumbaya, Prov. de Jujuy. Relaciones de la Sociedad Argentina de Antropología 7: 101-127.

FOX, A. M. y M. R. STRECKER, 1991. Pleistocene and modern snowline in the Central Andes (24-28 $8^{\circ}$ ). Bamberger Geographische Schriften 11: 169-182.

FRANCIS, P. W., M. GARDEWEG, C. F. RAMIREZ y D. A. ROTHERY, 1985. Catastrophic debris avalanche deposit of Socompa volcano, Northern Chile. Geology 13: 601-603.

FRITZ, P., C. H. SILVA, O. SUZUKI y E. SALATI, 1979. Isotope hydrology in Northern Chile. IAEA-SM-228/226: 525-543.

GRAF, K., 1992. Pollendiagramme aus den Anden. Eine Synthese zur Klimageschichte und Vegetationsentwicklung seit de letzten Eis. Schriftenreihe Physische Geographie 34: 1-138 h.

GROSJEAN, M., B. MESSERLI y H. SCHREIER, 1991. Seenhochstände, Bodenbildung und Vergletscherung im Altiplano Nordchile: Ein interdisziplinärer Beltrag zur Klimageschichte der Atacama. Erste Resultate. Bamberger Geographische Schriften 11: 99-108.

GROSJEAN, M. en prensa. Paleohydrology of the laguna Lejía (Northern Chile) and climatic implications for lateglacialtimes. Paleoclimatology, Paleoecology, Paleogeographie.

HASTENRATH, S. y J. E. KUTZBACH, 1985. Late Pleistocene climate and water budget of the south American Altiplano. Quaternary Research 24: 249-256.

HESSE, B., 1982a. Animal domestication and oscillating climates. Journal of Ethnobiology 2: 1-15.

1982b. Archaeological evidence for camelid exploitation in the Chilean Andes. Säugetierkundliche Mitteilungen 30: 201-211.

KESSLER, A., 1985. Zur Rekonstruktion von spätglaziales Klima und Wasserhaushalt auf demperuanisch-boliviaischen altiplano. Zeitschriftt für Gletscherkunde und Glaiologie 21: $107-114$.

1991. Zur Klimaentwicklung au dem Altiplano seit dem letzten Pluvial. Freiburger Geographische Hefte 32: 141-148.

LAUER, W. y W. ERLENBACH, 1987. Die tropischen Anden. Geökologische Raumgliederung und ihre Bedeutung für den Menschen. Geographische Rundschau 39 (2); 86-95. 
LLAGOSTERA, A., 1979. 9700 years of maritime subsistence on the Pacific: An analysis by means of bioindicators in the north of Chile. American Antiquity 44 (2): 309-324.

LYNCH, T., 1973. Harvest timing, transhumance and the process of domestication. American Anthropologist 75: 1254-1259.

1986. Climate change and human settlement around the Late Glacial laguna de Punta Negra, Northern Chile: The preliminary results. Geoarchaeology 1 (2): 145-162.

- 1990. Quaternary climate, environment and the human occupation of the South American Andes. Geoarchaeology 5 (3): 199-228.

LYNCH, T. F., R. GILLESPIE, J. A J. GOWLETT y R. E. M. HEDGES, 1985. Chronology of Guitarrero Cave, Perú. Science 229: 864-867.

LYNCH, T. F. y C. M. STEVENSON, 1992. Obsidian hydratation dating and temperature controls in the Punta Negra Región of Northern Chile. Quaternary Research 37: 117-867.

MARKGRAF, V., 1989. Paleoclimates in Central and South America since 18000 BP based on pollen and lake-level records. Quaternary Science Review 8: 1-24.

MESSERLI, B., M. GROSJEAN, G. BONANI, A. BÜRGI, M. A. GEYH, K. GRAF, K. RAMSEYER, H. ROMERO, U. SCHOTTETER, H. SCHREIER y M. VUILLE, 1993. Climate change and natural resource dynamics in the Atacama Altiplano. Mountain Research and Development 13 (2): 117-127.

MONTANE, J., 1972. Las evidencias del poblamiento temprano de Chile. Pumapunku 5: 40-53.

NUÑEZ, L., 1983. Paleoindian and Archaic cultural periods in the Arid and Semiarid Regions of Northern Chile. Advances in World Archaeology 2: 161-201.

1989. Hacia la producción de alimentos y la vida sedentaria. En Culturas de Chile. Prehistoria. Desde sus orígenes hasta los albores de la Conquista, J. Hidalgo, V. Schiappacasse, H. Niemeyer, C. Aldunate e I. Solimano (Eds.), pp. 81-105. Editorial Andrés Bello, Santiago.

1992. Ocupación arcaica en la Puna de Atacama: Secuencia, movilidad y cambio. En Prehistoria sudamericana. Nuevas perspectivas, B. Meggers (Ed.), pp. 283-307. Taraxacum, Washington D. C.

NUÑEZ, L. y C. SANTORO, 1988. Cazadores de la Puna Seca y Salada del Area Centro Sur Andina (norte de Chile). Estudios Atacameños 9: 11-60.
1990. Primeros poblamientos en el cono sur de América (XII-IX milenio AP). Revista de Arqueología Americana 1: 91-139.

NUÑEZ, L., J. VARELA, R. CASAMIQUELA, V. SCHIAPPACASSE, H. NIEMEYER y C. VILLAGRAN, 1992. Mastodont kill in Central Chile. Abstracts First World Conference on Prehistoric Mongoloid Dispersals, pp. 28. University of Tokio, Tokio.

NUÑEZ, L., J. VARELA, R. CASAMIQUELA y C. VILLAGRAN, 1993Ms. Reconstrucción multidisciplinaria de la secuencia prehistórica de Quereo (centro norte de Chile).

OCHSENIUS, C., 1986. La glaciación Puna durante el Wisconsin, deglaciación y máximo lacustre en la tradición WisconsinHoloceno y refugios de la megafauna postglaciales en la Puna y Desierto de Atacama. Revista de Geografía Norte Grande 13: 29-58.

PHILLIPPI, R., 1983? Noticias preliminares sobre los huesos fósiles de Ulloma. Anales de la Universidad de Chile 82 (9). Imprenta Cervantes, Santiago.

ROLLINS, H. B., J. B. RICHARDSON III y D. SANDWEISS, 1986. The birth of El Niño: Geoarchaeological evidence and implications. Geoarchaeology 1 (1): 3-15.

SCHREIER, H., M. GROSJEAN, L. LAVKULICH y B. MESSERLI, Ms. Paleosoils in the Atacama Altiplano: Indicators for Early Holocene climate change.

SELTZER, G. O., 1990. Recent glacial history and paleoclimate of the Peruvian-Bolivian Andes. Quaternary Science Review 9: $137-152$.

SINCLAIRE, C., 1985. Dos fechas radiocarbónicas del alero Chulqui, río Toconce: Noticia y comentario. Chungara 14: 71-79.

VEIT, H., 1991. Jungquartäre Relief-und Bodenentwicklung in der Hochkordillere im Einzungsgebiet des río Elqui, Nordchile, $30^{\circ}$ S. Bamberger Geographische Schriften 11: 81-97.

WIRRMANN, D. y L. F. DE OLIVEIRA, 1987. Low Holocene lake level (7700 to 3650 years ago) of lake Titicaca (Bolivia). Palaeogeography, Palaeoclimotology, Palaeoecology 59: 315-323.

YACOBACCIO, H., 1983. Explotación complementaria de recursos en sociedades cazadoras recolectoras surandinas. Cuadernos del Instituto Nacional de Antropología 10: 493-514. 